\title{
Téoros
}

Revue de recherche en tourisme

\section{Entretien avec le professeur Jean-Michel Hoerner}

\section{Alain A. Grenier}

Volume 28, numéro 2, 2009

URI : https://id.erudit.org/iderudit/1024816ar

DOI : https://doi.org/10.7202/1024816ar

Aller au sommaire du numéro

\section{Éditeur(s)}

Université du Québec à Montréal

\section{ISSN}

0712-8657 (imprimé)

1923-2705 (numérique)

Découvrir la revue

\section{Citer ce document}

Grenier, A. A. (2009). Entretien avec le professeur Jean-Michel Hoerner. Téoros, 28(2), 102-103. https://doi.org/10.7202/1024816ar d'utilisation que vous pouvez consulter en ligne.

https://apropos.erudit.org/fr/usagers/politique-dutilisation/ 


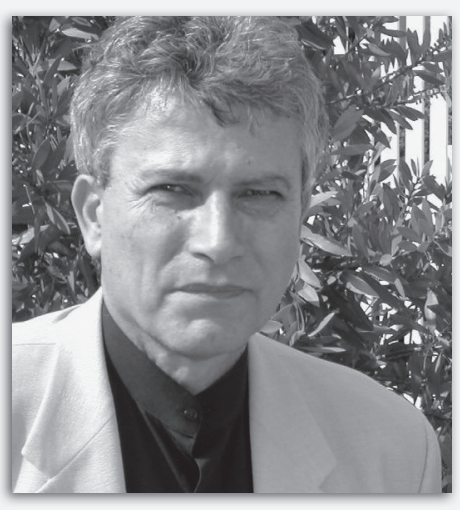

Le professeur Jean-Michel Hoerner est spécialiste de géopolitique et de tourisme à I'Université de Perpignan Via Domitia, en France. Ancien président de l'Université de Perpignan (1997-2002), le professeur Hoerner y a créé l'Institut universitaire professionnalisé des métiers du transport, de l'hôtellerie, du tourisme et des loisirs, qui a intégré la nouvelle Faculté de Sports, Tourisme, Hôtellerie Internationale, dont il est le doyen depuis 2002.

Propos recueillis par Alain A. Grenier, directeur et rédacteur en chef.

\section{Téoros : Parlez-nous de votre parcours académique. Comment} un étudiant devient-il éventuellement président d'université? Jean-Michel Hoerner : Je suis très atypique et je ne pensais pas du tout faire cette carrière. J'ai fait mon service militaire dans la coopération à Madagascar et j'y suis resté 18 ans. Mes amis, les professeurs Jean Cabot et Yves Lacoste, m'ont demandé de faire une thèse de doctorat, que j'ai soutenue en 1987, sur le sousdéveloppement dans le sud-ouest de Madagascar.

En 1989, j'ai été élu professeur à Perpignan, et comme j'étais toujours aussi atypique, je me suis retrouvé doyen de notre Faculté de droit, des sciences économiques, des lettres et des sciences humaines dès 1991. Puis, j'ai été élu président de l'Université en 1997. Mes nombreuses charges administratives anciennes et plus récentes (membre du bureau du Conseil scientifique de l'AUF, conseiller économique et social à Montpellier, etc.) ne m'ont pas empêché de poursuivre ma carrière de chercheur : 20 livres publiés et une centaine d'articles ou de communications. Bien sûr, outre la géopolitique (questions économiques, politique mondiale, Sud), je me consacre surtout au tourisme et à l'industrie touristique, et c'est pourquoi j'ai contribué à créer la «science du tourisme» en 2000.

\section{Téoros : Comment se porte l'étude du tourisme et du sport à l'université ?}

Professeur Hoerner : Nous avons chez nous plus de 1000 étudiants très motivés et bien encadrés dans ces cursus. Cependant, l'état de la recherche n'y est pas encore à la hauteur de nos ambitions. Les sciences sociales et humaines concernées ne prennent pas le tourisme au sérieux, et c'est notamment le cas des universités françaises. Nous sommes toutefois satisfaits que les entreprises touristiques, dont les groupes hôteliers, recrutent à Bac +5 (seulement à Bac +2 au début des années quatre-vingt-dix), ce qui nous conduit à valoriser au maximum l'innovation et la croissance de la productivité. Au demeurant, nous sommes pourtant loin du compte. En fait, nos sciences sociales s'intéressent au tourisme mais, selon moi, restent déconnectées des vraies problématiques. Combien, parmi elles, se préoccupent de l'évolution de l'industrie touristique dans la globalisation financière? Je regrette, par ailleurs, que le tourisme et le sport ne soient pas plus associés dans nos formations. Pourtant, jadis, nous avons formé de bons cadres intermédiaires (Bac +3 et 4 ), tels que des joueurs de rugby devenus professionnels et même un champion du monde du tir à l'arc. Aujourd'hui, de tels programmes sont sur la touche...

\section{Téoros : Pourquoi?}

Professeur Hoerner : Former les jeunes est une chose, mais les amener vers le marché du travail en est une autre. En sport, les emplois de managers sont réservés aux brillants retraités sportifs. Beaucoup d'entre eux, de haut niveau, sont ainsi recrutés sur leur renommée, aux dépens de leurs qualités de gestionnaires. Dans notre département, les Pyrénées-Orientales, chaque année, rien que pour le rugby, une vingtaine de joueurs quittent la compétition et trouvent souvent un emploi en raison de leur notoriété. Le sport étant une activité de spectacle, nous devrions être plus performants, mais nous avons peur de former des étudiants qui ne trouveraient aucun débouché en raison des mauvaises concurrences. Nous savons également que les centres touristiques sont demandeurs du meilleur encadrement sportif, ou que les établissements liés à la pratique sportive exigent de bons directeurs et organisateurs. L'industrie touristique ne peut pas faire cette économie.

Téoros : Vous mentionnez la discrimination, voire le mépris à l'égard de l'étude et de la recherche en tourisme en général. D'où vient-elle?

Professeur Hoerner : C'est un non-sens! Le tourisme international, à lui seul, produit plus de 900 milliards de dollars en recettes annuellement. Autre exemple, le Tour de France à lui seul attire 15 millions de spectateurs! Le tourisme est une réelle activité économique. On le mesure mal et les universités n'en prennent pas assez conscience.

Peut-être est-ce dû aux mauvaises définitions qu'on donne au tourisme, qui doit être une activité économique comme les autres. L'OMT [l'Organisation mondiale du tourisme] considère que le touriste a un statut : «le touriste est quelqu'un qui passe une nuit... ». Pour moi, le touriste n'a pas de statut. Ce n'est pas celui "qui passe une nuit». C'est déjà un consommateur de produits touristiques. On a besoin de concepts, certes, mais l'OMT fait voter les siens par des membres de l'ONU et non par des scientifiques comme le requiert toute science. Ce n'est pas sérieux. Les universitaires devraient faire évoluer les mentalités. 
Téoros : Le tourisme est un phénomène qui se conjugue tourisme ceci, tourisme cela. Comment le sport et le tourisme se marient-ils?

Professeur Hoerner : C'est logique de décliner le mot «tourisme », et, sur sa relation avec le sport, nous savons qu'il y a une quantité de métiers qui sont à cheval sur les deux domaines. J'ai évoqué l'importance des sportifs de haut niveau reconvertis et je me demande comment ils ne pensent pas à suivre les formations nécessaires à leurs nouveaux métiers. Un champion n'est pas forcément un manager! Je ne doute pas que certains fassent des efforts dans ce sens, mais ce n'est pas organisé. Cela pourrait cependant se faire avant le parcours de haut niveau des grands sportifs ou après, mais qui s'en soucie? Est-ce d'ailleurs l'attente des professionnels des spectacles sportifs? Un champion est considéré comme compétent, mais on se contente d'utiliser son nom plutôt que d'exiger qu'il soit mieux formé. Cela changera peut-être, mais l'association systématique entre sport et argent permet toujours de faire l'économie de la meilleure professionnalisation.

Téoros : Dans les publications anglo-saxonnes, on semble avoir réussi à délimiter un certains nombres de concepts, de perceptions. Dans la littérature française, le concept de tourisme de sports ou de tourisme sportif semble moins circonscrit.

Professeur Hoerner : J'ai souvent pensé que les anglophones sont en avance sur nous, c'est-à-dire les francophones (rire). II y a longtemps qu'on forme des professionnels d'encadrement du tourisme à Tallahassee, en Floride (1947), et même en Norvège, il y a une grande école hôtelière depuis 1912. En France, on a commencé à enseigner sérieusement le tourisme dans les établissements publics universitaires qu'à partir de 1991. On a donc beaucoup de retard. Vous savez, l'anglais est une langue obligatoire en tourisme, mais cela est entré dans nos mœurs il y a très peu de temps.

\section{Téoros : Selon vous, quels sont les grands problèmes} qui devraient mobiliser la connaissance du sport reliée au tourisme?

Professeur Hoerner : C'est une vaste question et je n'ai pas réponse à tout. Chacun sait pourtant, comme on l'a d'ailleurs évoqué, que le sport offre des spectacles presque touristiques et, en tout cas, ce n'est plus à démontrer pour les grands championnats du monde (football, rugby, basket, etc.), pour les Jeux olympiques, pour le Tour de France cycliste, etc. Des journaux, des revues, le Net, sont mobilisés. Or, les liens entre ces grands spectacles, qu'on pourrait multiplier à l'infini, et l'industrie touristique

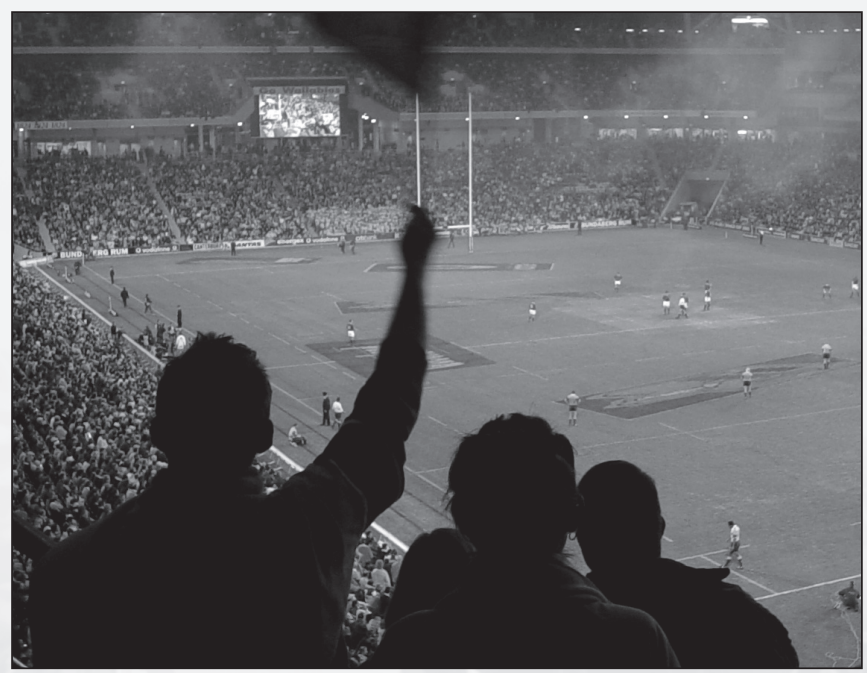

Le sport, activité de spectacle, fait déplacer les visiteurs par milliers (source : BJN, Stock.xchng).

ne sont pas assez développés. II ne s'agit pas de former les sportifs dans leur discipline, mais de leur permettre de bénéficier d'un public averti et structuré, ce qui requiert un certain savoirfaire. Combien y a-t-il d'organisateurs de voyage et d'agences de voyages spécialisés dans la pratique du tourisme sportif? A-t-on seulement pensé à développer des clubs de partisans sur la base des spectacles sportifs? Tout reste à faire et, naturellement, les formations universitaires pourraient y contribuer.

Téoros : Aux étudiants et étudiantes qui entreprennent leurs études en tourisme aujourd'hui, quel conseil donnez-vous? Quelle importance doit-on accorder aux études en tourisme? Professeur Hoerner : Il faut qu'ils s'affirment, ou plutôt qu'ils montrent leur spécificité. Je leur demande donc de ne jamais négliger la recherche. D'une part, les étudiants doivent atteindre les plus hauts niveaux de formation $(\mathrm{Bac}+5)$. D'autre part, les chercheurs ne doivent pas négliger les questions touristiques au sein même de leur domaine professionnel. Je dis souvent, et je le prouve, que le tourisme, notamment d'affaires, accompagne le système capitaliste depuis ses débuts en Europe, pendant le haut Moyen Âge. Aujourd'hui, le tourisme international est l'une des vitrines de la mondialisation. Qui, aujourd'hui, se soucie de ce phénomène économique et culturel? Je suis confiant, mais toujours inquiet. Finalement, le tourisme est trop considéré comme un ensemble de connaissances en marketing, en management, en informatique et en langues étrangères. C'est bien, mais c'est insuffisant. Sans oser l'avouer, je pense que la "science du tourisme » que je prône intègrera un coin de la philosophie. Je ne suis pas géopolitiste pour rien. 